Quelques Insectes du Pliocène supérieur du Comté de Durham, par M. Pierre Lessie.

Mistress Eleanor-M. Reid m'a fait l'honneur de me soumettre plusieurs débris d'Insectes qu'elle a recueillis dans les dépôts anciens de Castle Eden, sur la côte du Comté de Durham (Angleterre septentrionale). Ces dépôts, situés au-dessous du boulder clay, et par conséquent préglaciaires, sont rapprochés par Mistress Reid, au point de vue de leur àge, des couches de Tegelen (Limbourg hollandais). Ils dateraient du pliocène supérieur et seraient probablement contemporains de la période froide du crag de Norwich.

Les Insectes de Castle Eden sont représentés par des débris peu nombreux, généralement très fragmentaires. De ce fait, quelques uns échappent à toute identification. Ceux dont j’ai pu tirer parti appartiennent à dix espèces distinctes, dont neuf seulement peuvent donner prise à des déductions utiles.

Ces débris sont étudiés ci-dessous.

\title{
QRdRe des OR'THOPTÉRES.
}

\section{Forfigula auricularia L.}

Un pronotum presque entier, parfailement semblable à celui de la forme actuelle.

Espèce commune dans toute l'Europe et existant également dans la région méditerranéenne et dans l'Amérique du Nord. Elle est assez ubiquiste au point de vue de l'habitat.

\section{Ordre des COLÉoptìkes.}

2. Trechus amplicollis præglacialis, nov. subsp.

Fragment de l'élytre gauche comprenant à peu près les deux tiers basilaires. 
Les caractéristiques du débris peuvent être formulées comme il suit :

Teinte noire, à reflet très légèrement métallique pouvant résulter d'une altération d'un reflet irisé. 8 stries distinctes parallèles et sensiblement équidistantes el une striole scutellaire. Stries 6 et 7 beaucoup moins bien marquées que 1 à 5 , lesquelles sont fortement enfoncées, forlement et peu régulièrement ponctuées. Striole scutelaire coudée à angle obtus, canaliculiforme, graduellement atténuée d'avant en arrière, son bout antérieur marqué d'un pore sétigère situé dans l'axe du $2^{\mathrm{e}}$ interstrie; son

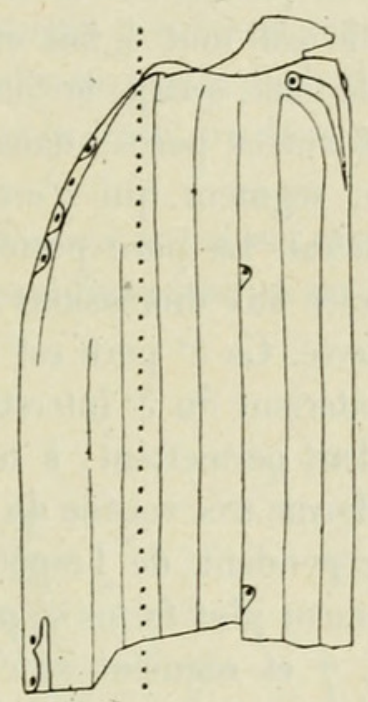

Fig. 1. - Trechus amplicollis pregglacialis.

Schéma de la topographie de l'élytre.

Le fragment comprend près des deux tiers basilaires de l'élytre gauche. On l'a supposé appliqué sur un plan, la partie externe ayant été rabattue dans le plan de la région dorsale autour du trait pointillé pris comme charnière.

L'épipleure manque. La ponctuation des stries n'a pas été figurée. Le rebord latéral s'arrète, en avant, un peu en dedans du bout antérieur de la $5^{\circ}$ strie; le trait qui parait la continuer vers la région scutellaire marque simplement le bord de la troncature basilaire de l'élytre. Des 6 pores visibles de la series umbilicet., 3 seulement s'ouvrent dans des fovéoles spéciales.

segment postérieur très rapproché du bord sutural. Stries 1 et 2 réunies en arc en avant; 3, 4, 5 rectilignes, paraillèles, libres à la base. Strie 3 marquée de 2 pores sétigères à son côté interne ( sur la longueur du frag. ment), le pore antérieur distant de la base de près du double de sa distance à la suture, le pore postérieur distant de l'antérieur de près du double de la distance de celui-ci à la base de l'élytre. Strie 5 un peu moins fortement marquée que les stries plus internes, atténuée en arrière. Stries 6 et 7 faiblement indiquées, presque effacées, 8 obsolète, à peine distincte, mais réapparaissant brusquement en arrière sous forme d'un cana- 
licule large et profond. Ce canalicule prend naissance à un niveau postérieur à celui du $2^{\circ}$ pore de la $3^{\circ}$ strie. Rebord latéral recourbé en avant dans la région humérale qu'il ne dépasse pas, en sorte qu'il n'existe pas de rebord le long de la base de l'élytre. Le bout antérieur du rebord marginal croise la $5^{\circ}$ strie qu'il limite en avant, et s'arrête à une faible distance en dedans de cette strie en se recourbant légèrement en arrière.

Series umbilicata conıprenant un groupe posthuméral de 4 pores, et deux pores rapprochés visibles au côté externe de la partie conservée du segment canaliculiforme de la $8^{\circ}$ strie. Les pores posthuméraux sont à peu près équidistants entre eux. Le pore antérieur est situé dans le prolongement de la $7^{\mathrm{e}}$ strie, laquelle est tout à fait effacée près de la base. Le $2^{\mathrm{e}}$ pore, le moins apparent de tous, occupe le fond du sillon subhuméral, sans être logé, comme les autres pores, dans une fovéole propre en forme de segment de cercle, segment qui n'est d'ailleurs qu'une simple dilatation du sillon subhuméral. Le pore postérieur du groupe posthuméral est le plus visible, gràce aux dimênsions plus grandes de la fovéole au centre de laquelle il s'ouvre. Ce $4^{\circ}$ pore est situé à un niveau à peine antérieur à celui du pore antérieur du $3^{\circ}$ interstrie.

Les caractères qui précèdent permettent, à mon avis, de rapporter le débris de Castle Eden à une forme très voisine du Trechus amplicollis Fairm. Le type pliocène diffère cependant de l'espèce actuelle par les stries internes des élytres notablement plus fortes et plus fortement ponctuées, par les stries externes $(6,7$ et segment antérieur de 8$)$ au contraire presque effacées, par la striole scutellaire plus longuement prolongée en en arrière et, par suite, plus rapprochée de la suture, et par la position des pores de la $3^{e}$ strie ${ }^{(1)}$.

Les pores posthuméraux de la series umbilicata présentent aussi quelques particularités, notamment la réduction du $2^{\circ}$ pore ou plutôt de ses dépendances, contrastant avec l'existence de fovéoles en segment circulaire encadrant les pores 1,3 et 4 .

Il n'est pas impossible que ces caraclères distinctifs aient une valeur spécifique. L'examen de nouveaux matériaux ou, à leur défaut, une étude comparative approfondie des Trechus vivants du groupe de l'amplicollis permettra sans doute de fixer ce point. Pour le moment, il y a avantage à faire figurer la forme de Castle Eden dans la nomenclature sous un nom qui rappelle son intime parenté avec l'espèce vivante.

Le Trechus amplicollis est aujourd'hui cantonné dans certains massifs montagneux de l'Europe moyenne, savoir : le plateau Central de la France

(1) Chez le T. amplicollis, le pore antérieur de la $3^{e}$ strie est situé à peu près à égale distance de la base de l'élytre et de la suture, à un niveau un peu antérieur à celui du $4^{\circ}$ pore de la series umbilicata. 
(Limoges, Saint-Sulpice-Laurière, le Mont-Dore, etc.), l'Istrie, les Sudètes, les Carpathes et la Transylvanie. Il fréquente les lieux humides et affectionne les terrains détrempés, notamment ceux où subsistent de petits creux d'eau (L. Bedel) ou qui sont situés au voisinage de la neige en fusion (P. de Vuillefroy). A ma connaissance, sa présence dans les l̂les Britanniques n'a jamais été constatée.

\section{Pterostichini (genus incertum, verisimiliter extinclum).}

Ce débris correspond à l'angle postérieur gauche du pronolum et aux régions voisines. Il comprend une partie du bord latéral et de la base du pronotum et permet d'étudier la sculpture de la région postérieure de cette partie du corps.

Le bord latéral, très faïblement arqué en arrière, fait un angle obtus avec la base et dénote un pronotum rétréci en arrière: ce bord est constitué

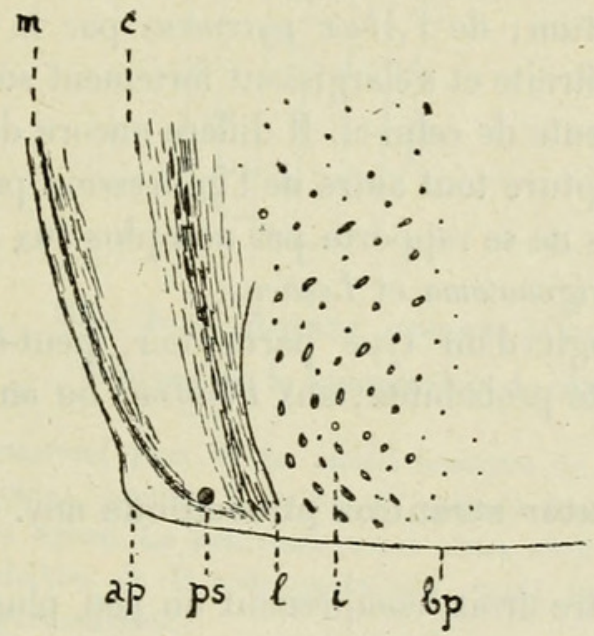

Fig. 2. - Pterostichini (genus incertum).

Angle postérieur gauche du pronotum et régions voisines.

$m$. bourrelet marginal externe dilaté sur l'angle postérieur, ap; $c$, eôte submarginale; $i$, impression postérieure, limitée en $l$ à son côté externe et marquée d'une sculpture spéciale; $b p$, bord postérieur du pronotum; $p s$, pore sétigère.

par une sorte d'ourlet épaissi, de largeur uniforme, au côté interne duquel s'étend, parallèlement à lui, un sillon large et assez vague, limité lui-même au dedans par une côte très large. An delà de cette côte, dans la direction de la ligne médiane, s'étend, sur les parties postérieures du disque du pronotum, une aire fortement sculptée et, en outre, impressionnée en arrière. Sa sculpture consiste en points arrondis assez gros et en traits courts disposés suivant des lignes concentriques assez distinctes. 
L'angle postérieur du pronotum est remarquable. Il est comme brièvement lobé, par suite de la dilatation de l'ourlet marginal à son bord externe, au sommel même de l'angł̣e. C'est à l'extrémilé postérieure de l'ourlet, un peu en dedans de l'angle poslérieur, que vient aboutir la large côte submarginale. Au point de jonction existe un gros pore sétigère. Le bord basilaire est simple, très légèrement arqué.

Tégument noir.

Ce débris appartient évidemment à un Féronien du type Pterostichus. On peut le rapprocher du Platysma nigrum Schaller, de l'Europe septentrionale et moyenne, du P. Escholtzi Germ., de Daourie, ou de l'Abax pyrenæus Dej., des Pyrénées. Il ne se rapporte toutefois à aucune de ces espèces. Il diffère du premier par la forme du sommet de l'angle postérieur du pronotum qui, chez le $P$. nigrum, présente une sorte de constriction préangulaire n'existant à aucun degré dans le fragment de Castle Eden. Il en diffère également par la longueur plus grande de la côte submarginale. Il se distingue du P. Escholtzi par la forme du sommet de l'angle postérieur du pronotum; de l'Abax pyrenaus par la marge latérale du pronotum bien plus étroite el s'élargissant fortement sur l'angle postérieur et par la forme différente de celui-ci. Il diffère encore des trois espèces qui précèdent par la sculpture tout autre de l'impression postérieure.

D'ailleurs le débris ne se rapporte pas non plus aux deux grands genres de l'Asie tropicale Trigonotoma et Lesticus.

Je pense qu'il s'agit d'un type particulier, peut-être disparu, mais apparenté, selon toute probabilité, aux Platysma ou aux Omaseus.

\section{Argutor strenuus pliocenicus nov. subsp.}

Fragment de l'élytre droit, comprenant un peu plus de la moitié basilaire.

Caractéristiques. - Région dorsale de l'élytre offrant 5 stries profondes, canaliculiformes, irrégulièrement ponctuées, les points étant effacés dan: la région siluée en arrière du niveau du pore discoüdal du $3^{\text {c }}$ interstrie. Ce pore est placé sur la $2^{\text {e }}$ strie, à son côté externe, à une distance de la base égale à 6 ou 7 fois celle qui sépare le pore de la suture. En arrière du pore sétigère discoïdal, le $3^{\mathrm{e}}$ interstrie devient un peu plus étroit que les interstries adjacents. Striole scutellaire reliée en arrière à la strie suturale; celte striole imponctuée ainsi que le segment adjacent de la strie suturale. Interstries presques plans, lisses et brillants, imponctués. Stries 6 et 7 obsciètes, très peu distinctes, 8 el 9 très forles, canaliculiformes, la dernière conliguë à la carène épipleurale dans toute la longueur du fragment. 


\section{- $393-$}

Series umbilicala comportant une série de 6 pores posthuméraux (les autres groupes de pores du $9^{\mathrm{e}}$ interstrie manquent). Les deux premiers pores sont peu développés et assez difficiles à distinguer par suite, semblet-il, d'une compression du débris dans celte région. Le second pore est précédé d'un léger renflement transverse. 3 ere très apparent, plus gros

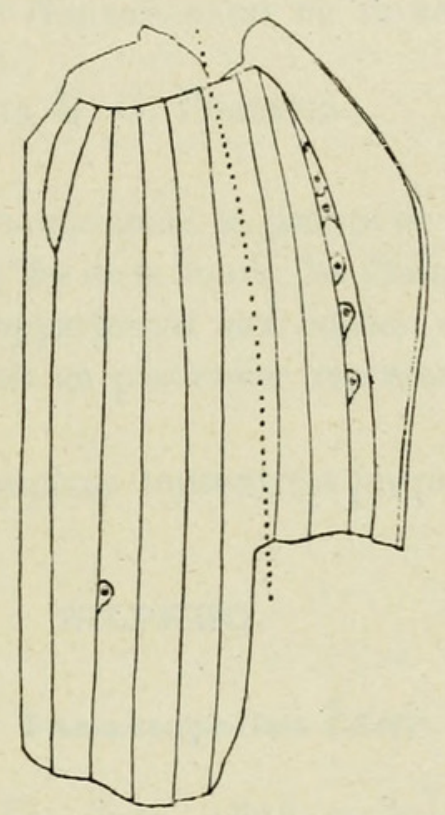

Fig. 3. - Argutor strenuus pliocenicus.

Schéma de la topographie de l'élytre.

Le fragment comprend plus de la moitié basilaire de l'élytre droit. Il est supposé appliqué sur un plan, comme il a été dit pour la figure 1. L'épipleure, ayant été conservé, a pu ètre figuré. La ponctuation des stries n'est pas indiquée.

Des six pores visibles de la series umbilicata, seuls les trois postérieurs s'ouvrent dans des fovéoles particulières.

que les précédents, occupant presque toute la largeur de la rainure subhumérale et précédé d'une tubérosité annexe bulboïde. C'est en arrière de ce $3^{\text {e }}$ pore que débute la $9^{\circ}$ strie. $4^{\circ}$ pore plus petit que le précédent, occupant le fond d'une fovéole semicordiforme. $5^{\mathrm{e}}$ pore aussi gros que le $3^{\circ}$, occupant également le fond d'une fovéole semicordiforme, celle-ci plus longue et plus large que la fovéole du $4^{\circ}$ pore. $6^{\mathrm{e}}$ pore écarté du $5^{\mathrm{C}}$ et moins gros que lui, logé également dans une fovéole. Épipleure large à la base, marqué d'une strie marginale le long de son bord libre.

Dans ce qu'ils ont d'essantiel, ces caractères sont ceux de l'Argutor strenuus Panz., Carabique répandu dans toute l'Europe et fréquentant les terrains froids; les bois humides, où il se tient sous les pierres, sous les fenilles et sous les mousses. 


\section{$-394-$}

La forme de Castle Eden diffère cependant de l'espèce vivante par ses stries élytrales beaucoup plus fortes, par les fovéoles réceptrices des pores de la series umbilicata plus grandes et plus profondes, par l'absence du pore antérieur du 3 interstrie.

Ici, comme dans le cas du Trechus pregglacialis, il y a intérêt à affecter à la forme fossile un nom rappelant ses affinités immédiates.

(A suivre.) 


\section{$2 \mathrm{BHL}$ Biodiversity Heritage Library}

1920. "Quelques insectes du pliocene superieur du comte de Durham." Bulletin du Muse

um national d'histoire naturelle 1920, 388-394. https://doi.org/10.5962/bhl.part.1206.

View This Item Online: https://www.biodiversitylibrary.org/item/27200 DOI: https://doi.org/10.5962/bhl.part.1206

Permalink: https://www.biodiversitylibrary.org/partpdf/1206

\section{Holding Institution}

New York Botanical Garden, LuEsther T. Mertz Library

\section{Sponsored by}

MSN

\section{Copyright \& Reuse}

Copyright Status: NOT_IN_COPYRIGHT

This document was created from content at the Biodiversity Heritage Library, the world's largest open access digital library for biodiversity literature and archives. Visit BHL at https://www.biodiversitylibrary.org. 